\title{
Obesity, Insulin Resistance, and Cardiovascular Disease
}

\author{
Gerald Reaven, Fahim Abbasi, and Tracey McLaughlin \\ School of Medicine, Stanford University, Stanford, California 94305
}

\begin{abstract}
The ability of insulin to stimulate glucose disposal varies more than six-fold in apparently healthy individuals. The one third of the population that is most insulin resistant is at greatly increased risk to develop cardiovascular disease (CVD), type 2 diabetes, hypertension, stroke, nonalcoholic fatty liver disease, polycystic ovary disease, and certain forms of cancer. Between 25-35\% of the variability in insulin action is related to being overweight. The importance of the adverse effects of excess adiposity is apparent in light of the evidence that more than half of the adult population in the United States is classified as being overweight/obese, as defined by a body mass index greater than $25.0 \mathrm{~kg} / \mathrm{m}^{2}$. The current epidemic of overweight/obesity is most-likely related to a combination of increased caloric intake and decreased energy expenditure. In either instance, the fact that CVD risk is increased as individuals gain weight emphasizes the gravity of the health care dilemma posed by the explosive increase in the prevalence of overweight/obesity in the population at large. Given the enormity of the problem, it is necessary to differentiate between the CVD risk related to obesity per $s e$, as distinct from the fact that the prevalence of insulin resistance and compensatory hyperinsulinemia are increased in overweight/obese individuals. Although the majority of individuals in the general population that can be considered insulin resistant are also overweight/obese, not all overweight/obese persons are insulin resistant. Furthermore, the cluster of abnormalities associated with insulin resistance - namely, glucose intolerance, hyperinsulinemia, dyslipidemia, and elevated plasma C-reactive protein concentrations - is limited to the subset of overweight/obese individuals that are also insulin resistant. Of greater clinical relevance is the fact that significant improvement in these metabolic abnormalities following weight loss is seen only in the subset of overweight/obese individuals that are also insulin resistant. In view of the large number of overweight/obese subjects at potential risk to be insulin resistant/hyperinsulinemic (and at increased CVD risk), and the difficulty in achieving weight loss, it seems essential to identify those overweight/obese individuals who are also insulin resistant and will benefit the most from weight loss, then target this population for the most-intensive efforts to bring about weight loss.
\end{abstract}

\section{Introduction}

Introduction of a specific immunoassay for quantifying plasma insulin concentrations (Yalow and Berson, 1960) had an enormous impact on understanding the relationship between obesity, insulin resistance, plasma insulin concentrations, type 2 diabetes, and cardiovascular disease (CVD). In that publication, Yalow and Berson pointed out that plasma immunoreactive insulin concentrations following an oral glucose challenge were as high, if not higher, in 
patients with what was then called "maturity-onset diabetes" than in a population with normal glucose tolerance. This observation led them to speculate that the tissues of patients with maturity-onset diabetes did not respond normally to insulin: in more-contemporary terms, they were insulin resistant. Shortly thereafter, papers began to appear that initiated a controversy concerning the relationship between obesity, insulin resistance, and hyperinsulinemia that continues to this day. Karam and colleagues, using a somewhat different insulin immunoassay (Karam et al., 1965), argued that plasma insulin concentrations were elevated in response to a glucose challenge only in patients with maturity-onset diabetes who were also obese. Based on these findings, they concluded that it was obesity, not maturity-onset diabetes, which was associated with insulin resistance. The suggestion by Karam and colleagues that obesity was associated with insulin resistance was consistent with the results of Rabinowitz and Zierler (1962), who had used the perfused forearm technique to provide direct evidence that insulin-stimulated glucose disposal was impaired in obese individuals. Although the observation that obesity was associated with insulin resistance did not necessarily mean that obesity was the sole cause of the defect in insulin action, the results of these and somewhat-similar studies have led to a commonly expressed view that insulin resistance, and its metabolic consequences, are simple manifestations of obesity.

An alternative view, and one that has been the focus of our research group for approximately 35 years, is that obesity is only one of several factors that modulate insulin action and by no means does insulin resistance equal obesity. The remainder of this chapter will summarize our efforts to address the relationship between obesity, insulin resistance, and CVD.

\section{Relationship Between Obesity, Plasma Insulin Concentration, and Type 2 Diabetes}

Our research group has published several papers that addressed the relationship between obesity, plasma insulin concentration, and type 2 diabetes. The results of these studies have been quite consistent in showing that plasma insulin concentrations in patients with type 2 diabetes are more closely associated with degree of hyperglycemia than with obesity. This section will discuss the results of three publications relevant to this issue.

Although the paper by Karam and coworkers (1965) was interpreted as showing that plasma insulin concentrations were high only in patients with type 2 diabetes who were also obese, the researchers did not control for the fact that the nonobese patients were significantly more hyperglycemic. Therefore, we (Reaven and Miller, 1968) determined the plasma glucose and insulin responses to oral glucose in 125 individuals, divided into four groups on the basis of progressive degrees of hyperglycemia: 1) normoglycemic; 2) impaired glucose 
tolerance; 3) mild diabetes (hyperglycemia); and 4) severe diabetes (hyperglycemia). Each of these four groups was further subdivided on the basis of degree of obesity into those who were less than $10 \%$ overweight, $10-30 \%$ overweight, or $30-50 \%$ overweight. The total integrated insulin responses of these experimental groups to an oral glucose challenge are shown in Figure 1, the legend of which defines the glucose levels of the four groups. It can be seen from these data that the total plasma integrated insulin response was somewhat higher than normal in the group with impaired tolerance, comparable to normal in patients classified as having mild diabetes, and somewhat lower in those with severe diabetes. These data showed that the insulin response to an oral glucose challenge varied considerably as a function of degree of hyperglycemia. Furthermore, when individuals were matched for plasma glucose concentration, differences in weight had very little effect on insulin response. Thus, the results of Karam and associates were essentially self-fulfilling in that they compared nonobese patients with relatively severe hyperglycemia to obese patients with lower levels of plasma glucose concentration. We would have come to the same confounded conclusion if we had compared plasma insulin concentrations without matching for both weight and degree of hyperglycemia.

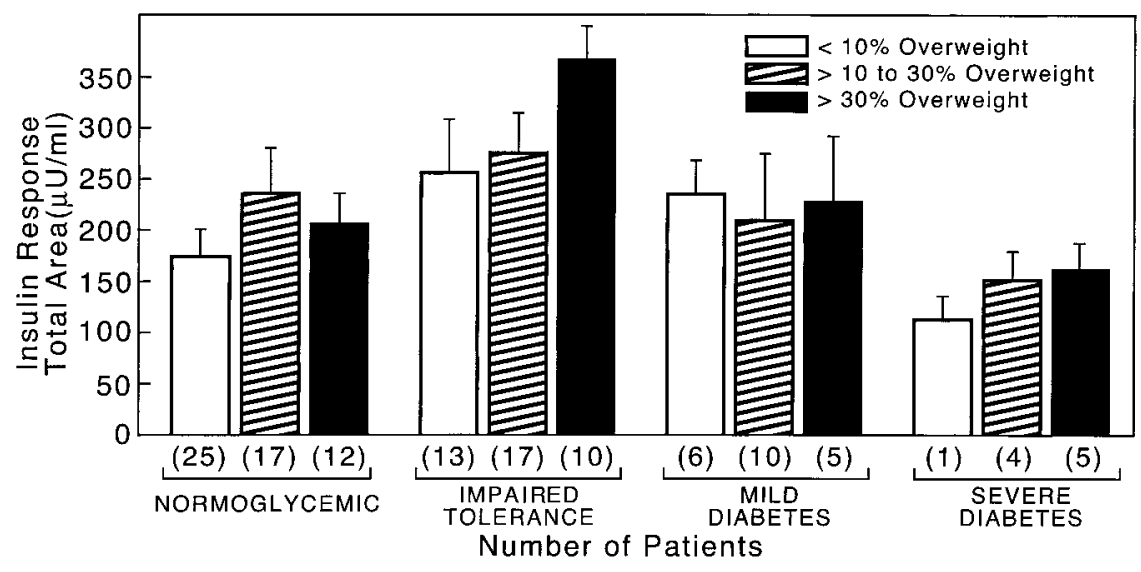

FIG. 1. Total integrated plasma insulin response to a 75-g oral glucose challenge in four groups of individuals differing in their degree of glucose tolerance. Within each glucose tolerance group, the individuals were subdivided further on the basis of their degree of obesity. Mean \pm fasting plasma glucose concentrations were $115 \pm 4$ and $217 \pm 13$ in the patients with "mild" and "severe" diabetes, respectively. The mean \pm plasma glucose concentrations 120 minutes after oral glucose challenge were $98 \pm 3,140 \pm 6,221 \pm 15$, and $411 \pm 22$ in the normoglycemic, impaired tolerance, "mild," and "severe" diabetes groups, respectively. [Reprinted with permission from Reaven GM, Miller R 1968 Study of the relationship between glucose and insulin responses to an oral glucose load in man. Diabetes 17:560-569.] 
Two other studies subsequently were performed in which the relationship between plasma insulin concentration and obesity was compared in patients with type 2 diabetes, always making sure to match subjects for obesity as well as degree of hyperglycemia. In the first study (Hollenbeck et al., 1984), we were able to demonstrate that both resistance to insulin-mediated glucose disposal and the plasma insulin response to an oral glucose challenge were comparable when obese and nonobese patients with type 2 diabetes were matched for degree of hyperglycemia. Finally, when questions were raised as to the specificity in earlier studies of the measurements of plasma insulin concentrations, we determined specific plasma insulin concentrations throughout the day in response to meals in both obese and nonobese individuals with varying degrees of glucose tolerance (Reaven et al., 1993). The results once again demonstrated that elevated plasma insulin concentrations in patients with glucose intolerance and/or type 2 diabetes varied as a function of degree of hyperglycemia, not body weight.

\section{Obesity and Insulin Resistance}

The observation that the hyperinsulinemia associated with glucose intolerance was not a simple function of obesity strongly suggested that this would also be true of the relationship between obesity and insulin resistance. This issue was first addressed in a study of 36 nondiabetic volunteers whose mean relative body weight (determined by Metropolitan Life Tables) was 1.21 (Olefsky et al., 1974). Insulin-mediated glucose disposal was quantified by the insulin suppression test, in which the steady-state plasma glucose (SSPG) concentration is determined at the end of a 180-minute infusion of insulin and glucose, with simultaneous suppression of endogenous insulin secretion (Shen et al., 1970; Greenfield et al., 1981; Pei et al., 1994). Under these conditions, the steady-state plasma insulin (SSPI) concentration at the end of the 180-minute infusion in all individuals is similar both in kind (exogenous) and amount. Since the amount of glucose infused is also similar in all subjects, the SSPG concentration is a direct measure of how effective insulin was in disposing of the infused glucose load: the higher the SSPG concentration, the more insulin resistant the individual. The results of this study indicated that the correlation coefficient ( $r$-value) between SSPG concentration and relative body weight was 0.46 . In other words, differences in adiposity accounted for approximately $25 \%$ of the variability in insulin-mediated glucose disposal in these overweight/obese individuals.

Although a relatively crude estimate of adiposity was used in this initial study, the results strongly suggested that being overweight increased the likelihood that an individual would be insulin resistant, although that the relationship was relatively modest in magnitude. Indeed, in a subsequent collaborative study performed in nondiabetic volunteers of both Pima Indian and European ancestry, the magnitude of the relationship between several estimates of adiposity and 
insulin-mediated glucose disposal was quite similar to our earlier findings (Bogardus et al., 1985). Parenthetically, it is important to note that, in the same study, we found that differences in physical fitness, as quantified by measurement of maximal aerobic capacity, were as powerful as variations in adiposity in modulation of insulin action. Finally, we recently completed a study of 314 healthy, nondidabetic, normotensive volunteers and the relationship between insulin resistance (SSPG) and body mass index (BMI) (Figure 2) (Abbasi et al., 2002). These data indicate that there is a statistically significant correlation between SSPG and BMI but, again, it can be seen that differences in BMI accounted for approximately $25 \%$ of the variability in insulin-mediated glucose disposal in this large population of healthy volunteers. Based upon these results, as well as evidence from several other studies published over the last 25 years, we believe it reasonable to conclude that obesity, along with physical inactivity, can account for approximately $50 \%$ of the variability in insulin-mediated glucose disposal in healthy, nondiabetic, normotensive individuals.

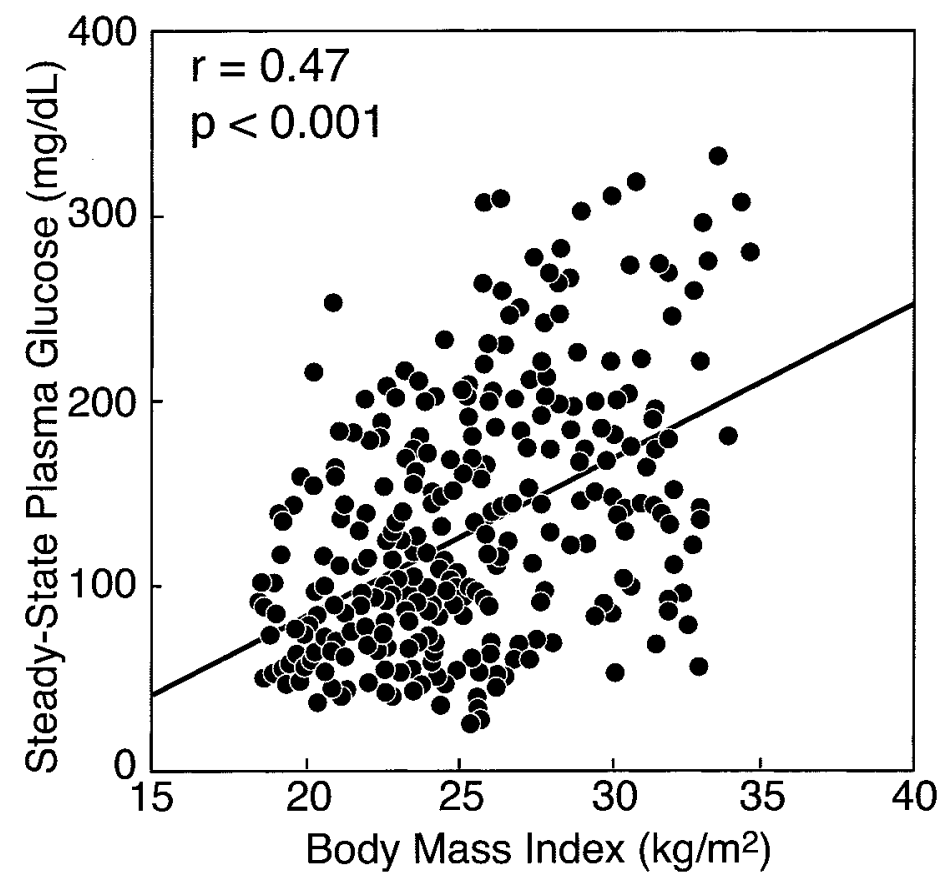

FIG. 2. Relationship between body mass index (BMI) and steady-state plasma glucose (SSPG) concentration in 314 healthy volunteers. [Reprinted with permission from Abbasi F, Brown BWB, Lamendola C, McLaughlin T, Reaven GM 2002 Relationship between obesity, insulin resistance, and coronary heart disease risk. J Am Coll Cardiol 40:937-944.] 


\section{Obesity, Insulin Resistance, and CVD Risk}

It can be seen from inspection of the data in Figure 2 that SSPG values varied several-fold in those individuals classified as being obese (BMI $>30.0$ $\mathrm{kg} / \mathrm{m}^{2}$ ). The obvious question that must be asked is whether CVD risk factors were similar in all the obese individuals in this study, or varied as a function of degree of insulin resistance? One way to approach this question is to calculate the correlation coefficients between each of the two variables, BMI and SSPG, and a number of CVD risk factors. The results, displayed in Table I, indicate that although almost all of the relationships depicted between the CVD risk factors measured and both BMI and SSPG were statistically significant, with differences in the magnitude of the correlation coefficients (r-values). Thus, BMI was more closely associated with age, systolic blood pressure (SBP), and concentrations of total and low-density lipoprotein cholesterol (LDL-C). In contrast, SSPG was more closely associated with diastolic blood pressure (DBP), triglyceride (TG), lower concentrations of high-density lipoprotein cholesterol (HDL-C), and higher plasma glucose and insulin responses to an oral glucose load. When these

TABLE I

Correlation Coefficients (r-values) Between Cardiovascular Disease Risk Factors, Body Mass Index (BMI), and Steady-state Plasma Glucose (SSPG)

\begin{tabular}{llc}
\hline & \multicolumn{2}{c}{ r Values } \\
\cline { 2 - 3 } Risk factors & BMI & SSPG \\
\hline Age & $0.19^{*}$ & $0.11^{* *}$ \\
SBP & 0.29 & $0.16^{*}$ \\
DBP & $0.14^{*}$ & 0.20 \\
Total cholesterol & 0.35 & 0.24 \\
TG & 0.44 & 0.51 \\
LDL-C & 0.36 & 0.22 \\
HDL-C & -0.38 & -0.41 \\
Glucose response & 0.31 & 0.57 \\
Insulin response & 0.38 & 0.63 \\
\hline
\end{tabular}

All r-values were statistically significant at the $\mathrm{p}<0.001$ level, with the exception of $* \mathrm{p}<0.05$ and $* * \mathrm{p}=0.16$. Abbreviations: SBP, systolic blood pressure; DBP, diastolic blood pressure; TG, triglyceride; LDL-C, low-density lipoprotein cholesterol; HDL-C, high-density lipoprotein cholesterol. Glucose and insulin responses are the total integrated area for the 180 minutes following a $75-\mathrm{g}$ oral glucose challenge. [Reprinted with permission from Abbasi F, Brown BWB, Lamendola C, McLaughlin T, Reaven GM 2002 Relationship between obesity, insulin resistance, and coronary heart disease risk. J Am Coll Cardiol 40:937-944.] 
same relationships were evaluated by multiple regression analysis, the results were quite similar. Thus, BMI was an independent predictor of SBP and total and LDL-C concentrations, whereas SSPG was an independent predictor of DBP and glucose response. Finally, although both BMI and SSPG were independent predictors of TG, HDL-C, and insulin concentrations, the magnitude of the relationship was much greater in the case of SSPG. Perhaps the relationship between BMI, SSPG, and CVD risk factors can be best understood by inspection of the data in Figures 3-5. To create these figures, we relied upon the results of a previous prospective study (Yip et al., 1998) that showed that $15 \%$ of apparently healthy individuals whose SSPG concentrations were in the upper tertile of the population at large developed manifest CVD over the approximately
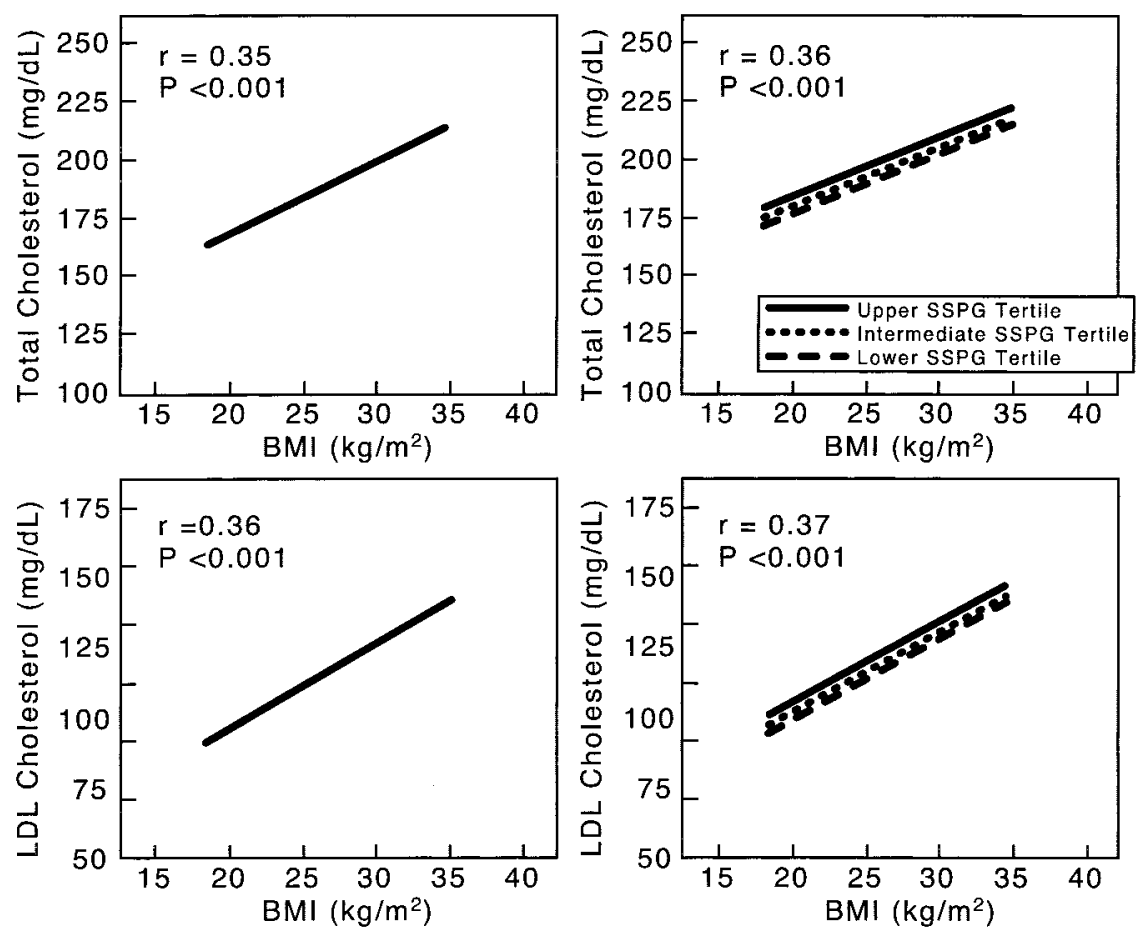

FIG. 3. The best-fit line describing the relationship between BMI and plasma concentrations of total cholesterol (upper panels) and low-density lipoprotein cholesterol (LDL-C) (lower panels) in 314 healthy volunteers. In each case, the panels on the left describe the relationship in the entire population, whereas the panels on the right portray the relationship when the group is divided into tertiles on the basis of their degree of insulin resistance (SSPG concentration). The r-values in the left panels are the correlation coefficients for the group as a whole, whereas the values on the right indicate the correlation coefficients when the relationships between BMI and the metabolic variables are adjusted for differences in degree of insulin resistance. 

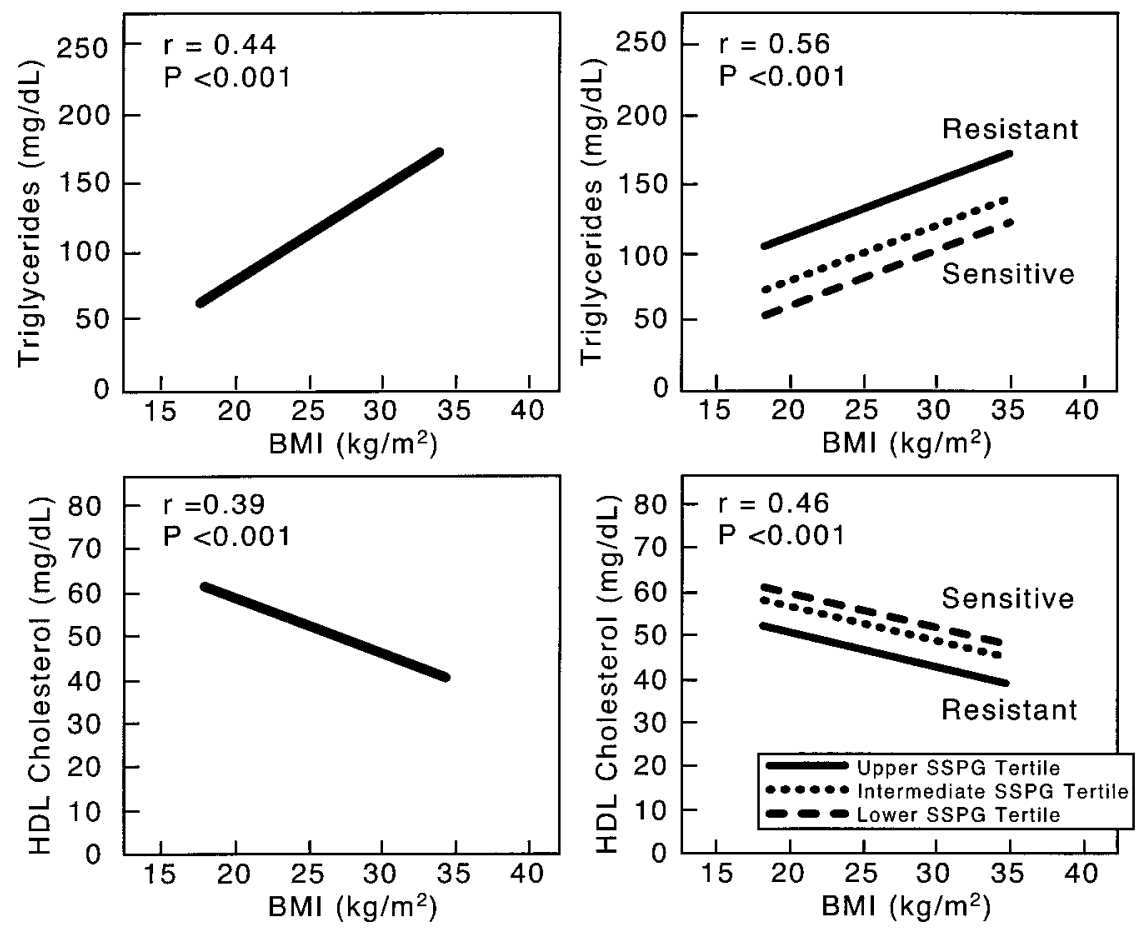

FIG. 4. The best-fit line describing the relationship between BMI and the concentrations of plasma triglyceride (TG) (upper panels) and low-density lipoprotein cholesterol (HDL-C) (lower panels) in 314 healthy volunteers. In each case, the panels on the left describe the relationship in the entire population, whereas the panels on the right portray the relationship when the group is divided into tertiles on the basis of their degree of insulin resistance (SSPG concentration). The r-values in the left panels are the correlation coefficients for the group as a whole, whereas the values on the right indicate the correlation coefficients when the relationships between BMI and the metabolic variables are adjusted for differences in degree of insulin resistance.

5-year period of observation. In contrast, none of those in the lower SSPG tertile displayed any evidence of CVD during this period. A subsequent prospective study (Facchini et al., 2001) confirmed the differences between the CVD risk of the upper and lower SSPG tertiles as well as pointed out that adverse clinical outcomes of those in the upper SSPG tertile were not limited to CVD. Based upon these findings, we operationally defined individuals whose SSPG concentrations fell within the upper tertile of the population at large as being insulin resistant (IR) and those with SSPG concentrations in the lowest SSPG tertile as being insulin sensitive (IS).

We then calculated the best-fit line for describing the relationship between BMI and the CVD variables in question in the entire population as well as 

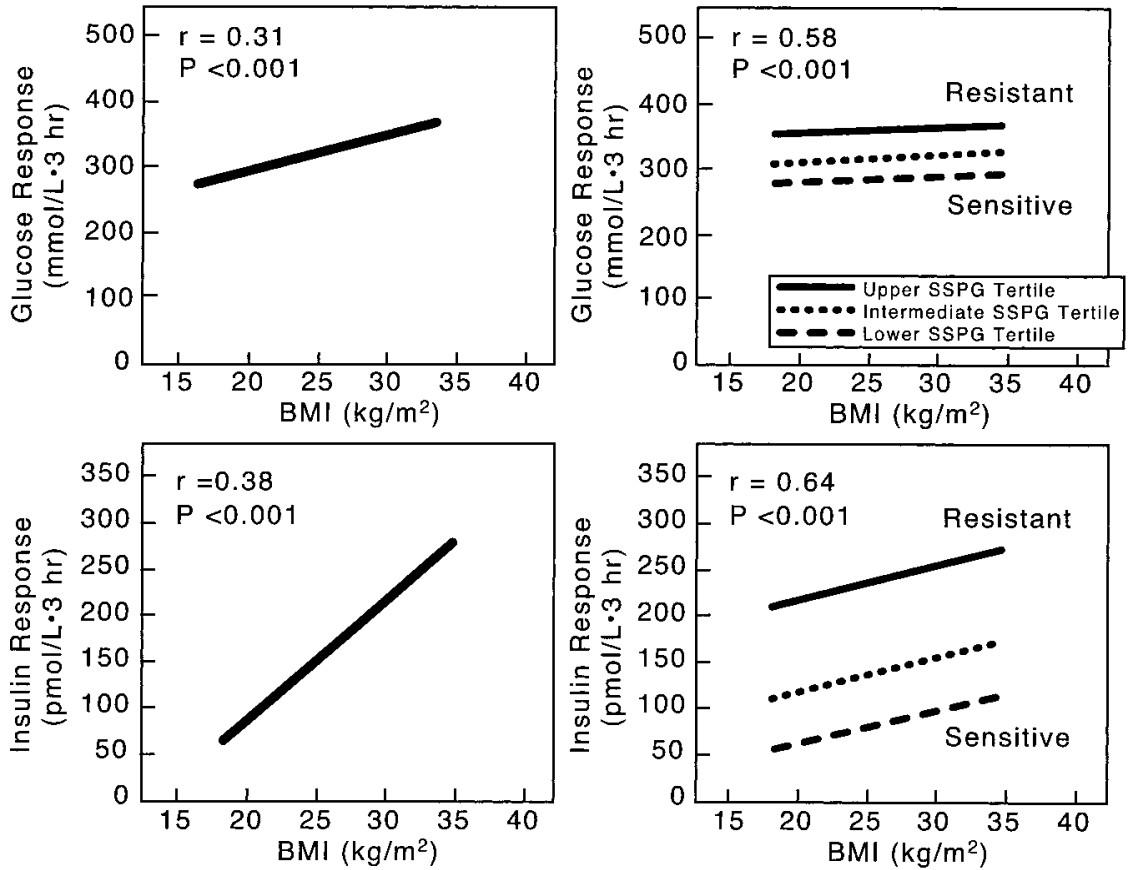

FIG. 5. The best-fit line describing the relationship between BMI and the plasma glucose (upper panels) and insulin responses to a 75-g oral glucose challenge (lower panels) in 314 healthy volunteers. In each case, the panels on the left describe the relationship between the entire population, whereas the panels on the right portray the relationship when the group is divided into tertiles on the basis of their degree of insulin resistance (SSPG concentration). The r-values in the left panels are the correlation coefficients for the group as a whole, whereas the values on the right indicate the correlation coefficients when the relationships between BMI and the metabolic variables are adjusted for differences in degree of insulin resistance.

separately for individuals in the IR, IS, and intermediate SSPG tertiles. This approach was used to create the relationships shown in Figures 3-5 between BMI, SSPG, and CVD risk factor. In each figure, the two left panels illustrate the relationship between BMI and the specific CVD risk factor for the entire population, whereas the right panels display the best-fit line between BMI and the CVD risk factor for the individuals in the three SSPG tertiles. If we focus on Figure 3, it is apparent from these data that the relationship between BMI and total and LDL-C concentrations seen in the entire group (left panels) does not vary as a function of SSPG tertile (right panels). In other words, at any given $\mathrm{BMI}$, the total and LDL-C concentration were relatively similar in IR and IS individuals. 
In contrast, the data in Figures 4 and 5 dramatically illustrate that the relationship between BMI and plasma TG and HDL-C concentrations (Figure 4) and plasma glucose and insulin concentrations in response to the oral glucose challenge (Figure 5) vary substantially as a function of insulin-mediated glucose disposal rates. More specifically, at any give BMI, the plasma TG concentrations are much higher and the HDL-C concentrations are much lower in the IR as compared to the IS tertile. Similarly, plasma glucose and insulin concentrations following an oral glucose challenge are two to three times higher in IR as compared to IS individuals.

Based upon these results, it is obvious that not all overweight/obese individuals are insulin resistant and that although insulin resistance and the CVD risk factors measured tend to increase as individuals get heavier, it is those overweight/obese individuals who are also insulin resistant that are at greatest CVD risk.

\section{Does Weight Loss Decrease CVD Risk?}

The studies to be discussed in this section fall into two categories. To begin with, it is necessary to address the issue of whether or not insulin resistance and/or compensatory hyperinsulinemia have any effect on the ability of overweight/obese individuals to lose weight. We will then discuss the improvement in CVD risk factors associated with weight loss.

\section{A. RELATIONSHIP BETWEEN INSULIN RESISTANCE AND ABILITY TO LOSE WEIGHT}

There appears to be considerable popular acceptance of the view, aggressively trumpeted in several popular diet books, that all overweight/obese individuals are insulin resistant and that it is their insulin resistance that prevents them from losing weight. Put more bluntly, this view states that the daylong hyperinsulinemia that permits insulin-resistant individuals to maintain normal, or near-normal, glucose tolerance prevents effective weight loss in overweight/ obese individuals. In our first effort to address this issue (McLaughlin et al., 1999), we performed SSPG concentrations in a series of obese individuals in apparently good health. They were then placed on a calorie-restricted diet and followed for 2 months.

The relationship between their degree of insulin resistance (SSPG concentration) and daylong insulin response to standard test meals before the period of weight loss is shown in Figure 6. It can be seen from this figure that the concentrations of both SSPG (two top panels) and plasma insulin (two lower panels) varied widely in the volunteers in this study. However, it is apparent from the data in the top two panels that there was absolutely no correlation between 

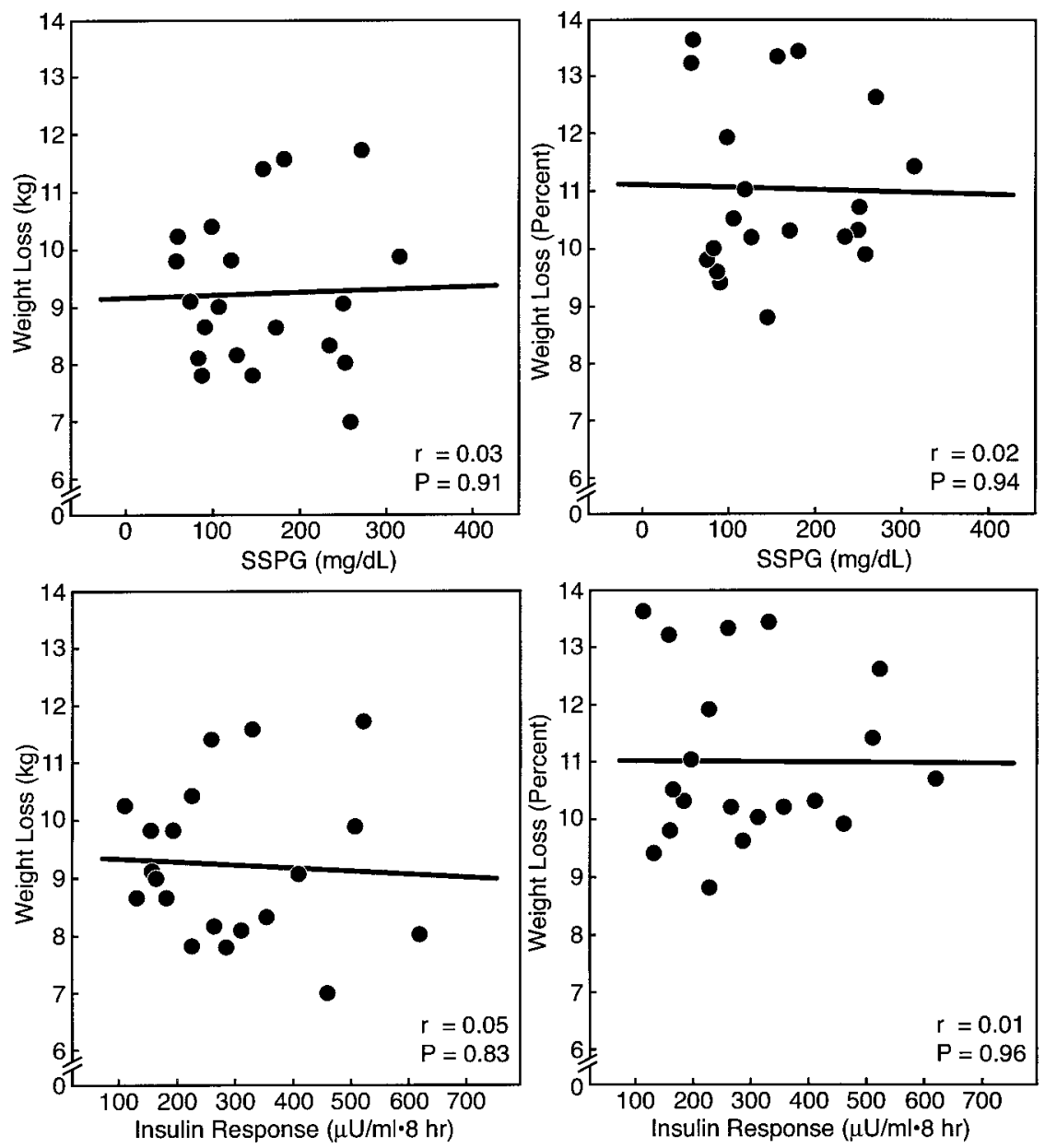

FIG. 6. The relationship between weight loss in response to 2 months of a calorie-restricted diet and the baseline SSPG concentration (upper panels) and daylong integrated plasma insulin response to breakfast and lunch (lower panels). [Reprinted with permission from McLaughlin T, Abbasi F, Carantoni M, Schaaf P, Reaven GM 1999 Differences in insulin resistance do not predict weight loss in response to hypocaloric diets in healthy obese women. J Clin Endocrinol Metab 84:578-581. Copyright The Endocrine Society.]

baseline SSPG concentration and weight loss. The data in the two lower panels indicate that there was also no relationship between baseline daylong insulin concentrations and ability to lose weight. To express it more succinctly, the obese individuals in this study lost weight as effectively, or ineffectively, irrespective of their degree of insulin resistance or daylong hyperinsulinemia. 
Although the results displayed in Figure 6 provided evidence that the ability of obese subjects to lose weight in response to calorie-restricted diets was independent of differences in SSPG and daylong insulin concentrations, this conclusion could be criticized on two counts. In the first place, the period of weight loss was only 2 months long. Because obesity usually results from slow but cumulative weight gain, the clinical significance of the study could be questioned. Second, our weight-loss protocol was highly controlled, relying primarily on a liquid nutritional formula rather than "real" food. Thus, while the experimental approach may have been useful in attempting to eliminate the possible confounding effect of varied caloric intake between subjects, it could be argued that it had little relevance to the manner in which weight-loss programs usually are carried out. Thus, a second study was initiated to extend our earlier findings and the protocol was amended to the address the two potential problems just outlined (McLaughlin et al., 2001). Specifically, the period of weight loss was extended to 4 months and the diet intervention was limited to nutritional advice, with all foods prepared by the subjects at home. In addition, to maximize potential for weight loss, the dietary advice was supplemented by administration of the appetite suppressant sibutramine.

The BMI of the volunteers enrolled in this study ranged between 30.0 and $36.0 \mathrm{~kg} / \mathrm{m}^{2}$. They were subdivided at baseline into IR (SSPG $\left.=219 \pm 7 \mathrm{mg} / \mathrm{dL}\right)$ and IS (SSPG $=69 \pm 6 \mathrm{mg} / \mathrm{dL}$ ) subgroups. The baseline weight of the two groups was similar ( $87 \pm 2 \mathrm{~kg}$ vs. $84 \pm 2 \mathrm{~kg}$ ) and there was no difference in the amount of weight loss after 4 months of a calorie-restricted diet in the IR and IS subgroups $(8.6 \pm 1.3 \mathrm{~kg}$ vs. $7.9 \pm 1.4 \mathrm{~kg})$.

Thus, doubling the weight-loss period and having the participants prepare all their own food did not change the overall conclusion that differences in baseline values of insulin-mediated glucose disposal and circulating insulin concentrations have little, if any, effect on the ability of an individual to lose weight.

\section{B. EFFECT OF WEIGHT LOSS ON CVD RISK FACTORS}

Although differences in insulin-mediated glucose disposal do not affect the ability of obese individuals to lose weight in response to a calorie-restricted diet, they certainly modify the benefits associated with weight loss. For example, Figure 7 contains measurements of body weight and SSPG concentrations before and after weight loss in 40 obese women, divided into IR and IS subgroups (McLaughlin et al., 2002). The two groups were not different in terms of age, weight, BMI, or waist circumference at baseline. By selection, the SSPG concentrations were higher in the IR group ( $228 \pm 8$ vs. $76 \pm 5 \mathrm{mg} / \mathrm{dL})$. The results in Figure 7 (left panel) again show that differences in insulin resistance did not affect weight loss. Weight decreased by a comparable amount $(8.7 \pm 0.9$ vs. $8.4 \pm 0.8 \mathrm{~kg}$ ) and to a similar final value in both groups. SSPG concentration 

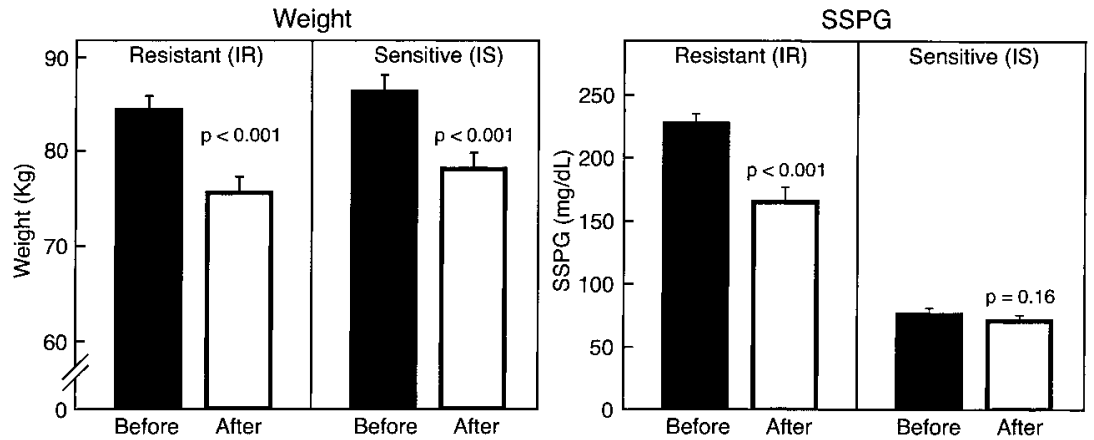

FIG. 7. Weight (left panels) and SSPG concentrations (right panels) before and after 4 months of a calorie-restricted diet in equally obese individuals, subdivided into insulin-resistant (IR) and insulin-sensitive (IS) subgroups on the basis of their SSPG concentrations at baseline. [Reprinted with permission from McLaughlin T, Abbasi F, Lamendola C, Liang L, Reaven G, Schaaf P, Reaven P 2002 Differentiation between obesity and insulin resistance in the association with C-reactive protein. Circulation 106:2908-2912.]

decreased significantly (right panel) to $165 \pm 12 \mathrm{mg} / \mathrm{dl}(\mathrm{p}<0.001)$ with weight loss in the IR group, although this value was still greater than the post-weight loss value in the IS group $(75 \pm 5 \mathrm{mg} / \mathrm{dl}, \mathrm{p}<0.001)$. SSPG concentration did not change significantly in the IS group with weight loss.

Daylong plasma glucose and insulin concentrations for both groups, before and after weight loss, are shown in Figure 8. Daylong plasma glucose concentrations (top panel) were significantly higher at baseline in the IR group than in the IS group $(p=0.005)$. In addition, plasma glucose concentrations declined significantly $(\mathrm{p}<0.001)$ with weight loss in the IR group. The daylong response was no longer significantly higher than in the IS group $(p=0.29)$. In contrast, daylong plasma glucose responses were essentially the same in the IS group, before and after weight loss.

The IR group had significantly greater daylong plasma insulin concentrations (Figure 8 , lower panel) than the IS group at baseline $(\mathrm{p}<0.005)$. Furthermore, although daylong integrated insulin concentrations were significantly lower following weight loss $(\mathrm{p}=0.01)$ in the IR group, it should be emphasized that the post-weight loss daylong insulin response in this group was still elevated, as compared with the post-weight loss daylong insulin response in the IS group ( $\mathrm{p}>0.001)$. Finally, daylong insulin concentration curves were similar before and after weight loss in the IS group.

In addition to the higher daylong plasma glucose and insulin concentration, we had shown in an earlier study that insulin-resistant women had higher plasma TG and lower HDL-C concentrations as well as higher plasma concentrations of plasminogen activator inhibitor-1 (Abbasi et al., 1999). Given this cluster of 


\section{Glucose}
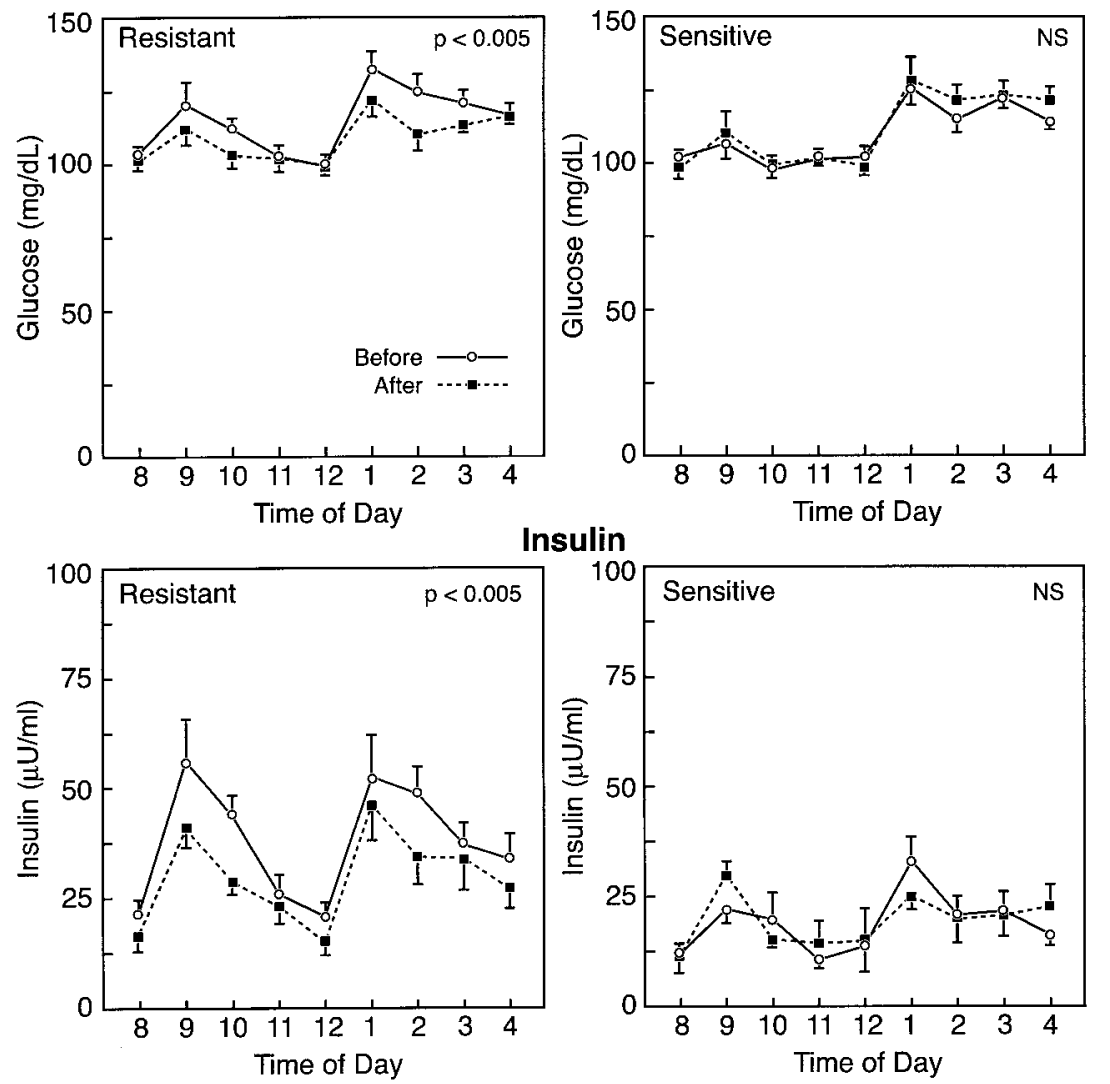

FIG. 8. Daylong plasma glucose (upper panels) and insulin responses (lower panels) before and after weight loss in obese individuals subdivided into IR and IS subgroups on the basis of their SSPG concentrations at baseline. [Reprinted with permission from McLaughlin T, Abbasi F, Lamendola C, Liang L, Reaven G, Schaaf P, Reaven P 2002 Differentiation between obesity and insulin resistance in the association with C-reactive protein. Circulation 106:2908-2912.]

abnormalities known to increase CVD risk, we also compared concentrations of high-sensitivity C-reactive protein (CRP) in the IR and IS groups of obese women. These results are seen in Figure 9, which presents CRP concentrations of the two groups, before and after weight loss. Baseline CRP concentrations were significantly higher in the IR group than in the IS group $(0.39 \pm 0.08$ vs. $0.12 \pm 0.03 \mathrm{mg} / \mathrm{dL}, \mathrm{p}=0.001)$. Furthermore, whereas plasma CRP concentrations were significantly lower after weight loss in the IR group $(p=0.04)$, there was no decline in CRP concentrations in the IS group. Despite the fall in CRP concentrations with weight loss in the IR group, the post-weight loss CRP 


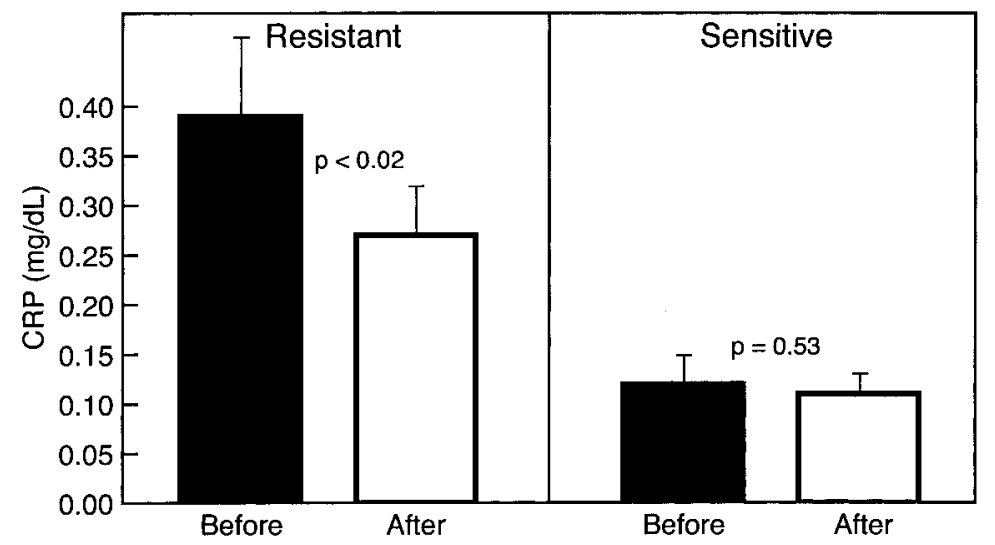

FIG. 9. C-reactive protein (CRP) concentrations before and after weight loss in obese individuals divided into IR and IS subgroups on the basis of their SSPG concentrations at baseline. [Reprinted with permission from McLaughlin T, Abbasi F, Lamendola C, Liang L, Reaven G, Schaaf P, Reaven P 2002 Differentiation between obesity and insulin resistance in the association with C-reactive protein. Circulation 106:2908-2912.]

concentrations were still higher in the IR group than in the IS group $(0.27 \pm 0.05$ vs. $0.11 \pm 0.02, \mathrm{p}=0.008$ ).

\section{Conclusions}

Recent reports (Kuczmarski et al., 1997) indicate that more than 50\% of the U.S. population is overweight (BMI $>25.0 \mathrm{~kg} / \mathrm{m}^{2}$ ), with approximately $20 \%$ designated as obese $\left(\mathrm{BMI}>30 \mathrm{~kg} / \mathrm{m}^{2}\right)$. The disease-related implications of this epidemic have received an enormous amount of publicity in the popular media but public awareness of the untoward effects of excess weight has not led to an effective approach to dealing with the dilemma. The gravity of the problem is accentuated in light of the report that only about half of the physicians polled provided weight-loss counseling (Galuska et al., 1999) and that pharmacological treatment of weight loss is not being used appropriately in overweight individuals (Khan et al., 2001).

Given the importance of excess adiposity as increasing risk of CVD, type 2 diabetes, and hypertension (West and Kalbfleisch, 1971; Havlik et al., 1983; Rimm et al., 1995), and the combination of an increase in the prevalence of overweight/obesity and a health care system unprepared to deal with this situation, it is essential that considerable thought be given as to how to best address this dilemma. In this context, it must be emphasized that CVD, type 2 diabetes, and hypertension are characterized by resistance to insulin-mediated 
glucose disposal (Reaven, 1988,2001) and that insulin resistance, as well as the compensatory hyperinsulinemia associated with insulin resistance, have been shown to be independent predictors of all three clinical syndromes (Yip et al., 1998; Zavaroni et al., 1999; Facchini et al., 2001). It has been apparent for many years that overweight/obese individuals tend to be insulin resistant and become more insulin sensitive with weight loss (Olefsky et al., 1974). In light of these observations, it seems reasonable to suggest that insulin resistance is the link between overweight/obesity and the adverse clinical syndromes related to excess adiposity. Based on this fundamental premise, our research group has performed a series of studies over the past several years in an effort to provide insights into the nature of this relationship. The evidence summarized in this review has shown that the more overweight an individual is, the more likely he/she will be insulin resistant and at increased risk to develop all the abnormalities associated with this defect in insulin action. However, we do not believe that all overweight/ obese individuals are insulin resistant, any more than all IR individuals are overweight/obese. More importantly, there is compelling evidence that CVD risk factors are present to a significantly greater degree in the subset of overweight/ obese individuals that are also insulin resistant. Not surprisingly, we have also demonstrated that an improvement in CVD risk factors with weight loss occurs to a significantly greater degree in those overweight/obese individuals who were also insulin resistant at baseline. In view of the ineffectiveness of current clinical approaches to weight loss, it seems necessary to recognize that not all overweight/obese individuals are at equal risk to develop CVD and that it is clinically useful to identify those at highest risk. If this is done, intense efforts at weight control can be brought to bear on those who not only need it the most but also have the most to gain by losing weight.

\section{REFERENCES}

Abbasi F, McLaughlin T, Lamendola C, Lipinska I, Tofler G, Reaven GM 1999 Comparison of plasminogen activator inhibitor-1 concentration in insulin-resistant versus insulin-sensitive healthy women. Arterioscler Thromb Vasc Biol 19:2818-2821

Abbasi F, Brown BWB, Lamendola C, McLaughlin T, Reaven GM 2002 Relationship between obesity, insulin resistance, and coronary heart disease risk. J Am Coll Cardiol 40:937-994

Bogardus C, Lillioja S, Mott DM, Hollenbeck C, Reaven, GM 1985 Relationship between degree of obesity and in vivo insulin action in man. Am J Physiol 248(Endocr Metab 11):E286-E291

Facchini FS, Hua N, Abbasi F, Reaven GM 2001 Insulin resistance as a predictor of age-related diseases. J Clin Endocrinol Metab 86:3574-3578

Galuska DA, Will JC, Serdula MK, Ford ES 1999 Are health care professionals advising obese patients to lose weight? JAMA 282:1576-1578

Greenfield MS, Doberne L, Kraemer FB, Tobey TA, Reaven GM 1981 Assessment of insulin resistance with the insulin suppression test and the euglycemic clamp. Diabetes 30:387-392

Havlik RJ, Hubert HB, Fabsitz RR, Feinleib M 1983 Weight and hypertension. Ann Intern Med 98:855-854 
Hollenbeck CB, Chen YD-I, Reaven GM 1984 A comparison of the relative effects of obesity and non-insulin-dependent diabetes mellitus on in vivo insulin-stimulated glucose utilization. Diabetes 33:622-626

Karam JH, Pavlatos FC, Grodsky GM, Forsham PH 1965 Critical factors in excessive seruminsulin response to glucose. Lancet 1:286-289

Khan LK, Serdula MK, Bowman BA, Williamson DF 2001 Use of prescription weight loss pills among US adults in 1996-1998. Ann Intern Med 134:282-286

Kuczmarski RJ, Carroll MD, Flegal KM, Troiano RP 1997 Varying body mass index cutoff points to describe overweight prevalence among US adults: NHANES III (1988 to 1994). Obesity Res 5:542-548

McLaughlin T, Abbasi F, Carantoni M, Schaaf P, Reaven GM 1999 Differences in insulin resistance do not predict weight loss in response to hypocaloric diets in healthy obese women. J Clin Endocrinol Metab 84:578-581

McLaughlinT, Abbasi F, Kim, H-S, Lamendola C, Schaaf P, Reaven GM 2001 Relationship between insulin resistance, weight loss, and coronary heart disease risk in healthy, obese women. Metabolism 50:795-800

McLaughlin T, Abbasi F, Lamendola C, Liang L, Reaven G, Schaaf P, Reaven P 2002 Differentiation between obesity and insulin resistance in the association with C-reactive protein. Circulation 106:2908-2912

Olefsky JM, Reaven GM, Farquhar JW 1974 Effects of weight reduction on obesity: studies of carbohydrate and lipid metabolism. J Clin Invest 53:64-76

Pei D, Jones CNO, Bhargava R, Chen YD-I, Reaven GM 1994 Evaluation of octreotide to assess insulin-mediated glucose disposal by the insulin suppression test. Diabetologia 37:843-845

Rabinowitz D, Zierler KJ 1962 Forearm metabolism in obesity and its response to intra-arterial insulin. Characterization of insulin resistance and evidence for adaptive hyperinsulinemia. J Clin Invest 41:2173-2181

Reaven GM 1988 Role of insulin resistance in human disease. Diabetes 37:1595-1607

Reaven GM 2001 Insulin resistance, compensatory hyperinsulinemia, and coronary heart disease: syndrome X revisited. In: Jefferson LS, Cherrington AD, eds. Handbook of Physiology, vol II, section 7. Oxford, UK: Oxford University Press; 1169-1197

Reaven GM, Miller R 1968 Study of the relationship between glucose and insulin responses to an oral glucose load in man. Diabetes 17:560-569

Reaven GM, Chen YD-I, Hollenbeck CB, Sheu WW-H, Ostrega D, Polonsky KS 1993 Plasma insulin, C-peptide, and proinsulin concentrations in obese and nonobese individuals with varying degrees of glucose tolerance. J Clin Endocrinol Metab 76:44-48

Rimm EB, Stampfer MJ, Giovannucci E, et al. 1995 Body size and fat distribution as predictors of coronary heart disease among middle-aged and older US men. Am J Epidemiol 141:1117-1127

Shen S-W, Reaven GM, Farquhar JW 1970 Comparison of impedance to insulin mediated glucose uptake in normal and diabetic subjects. J Clin Invest 49:2151-2160

West KM, Kalbfleisch JM 1971 Influence of nutritional factors on prevalence of diabetes. Diabetes 20:99-108

Yalow RS, Berson SA 1960 Immunoassay of endogenous insulin in man. J Clin Invest 39:1157-1175

Yip J, Facchini FS, Reaven GM 1998 Resistance to insulin-mediated glucose disposal as a predictor of cardiovascular disease. J Clin Endocrinol Metab 83:2773-2776

Zavaroni I, Bonini L, Gasparini P, Barilli AL, Zuccarelli A, Dall'Aglio E, et al. 1999 Hyperinsulinemia in a normal population as a predictor of non-insulin-dependent diabetes mellitus, hypertension, and coronary heart disease: the Barilla factory revisited. Metabolism 48:989-994 